\title{
Femtosecond pulse shaping via engineered nonlinear photonic crystals
}

\author{
U.K. Sapaev ${ }^{1}$, V.E. Eshniyazov${ }^{1}$, B. Kh. Eshchanov ${ }^{1}$ D. B. Yusupov ${ }^{2}$ \\ ${ }^{1}$ Department of Physics, National University of Uzbekistan, named after M. Ulugbek, \\ 100174 Tashkent, Uzbekistan \\ ${ }^{2}$ Tashkent State Technical University, Universitetskaya ul. 2 Tashkent, 100095 Uzbekistan \\ usapaev@gmail.com
}

PACS 42.65.-ky, 42.79.Nv

DOI 10.17586/2220-8054-2015-6-2-244-248

\begin{abstract}
Non-stationary second harmonic generation by femtosecond pulses, taking into account both group velocity mismatch and dispersion in nonlinear photonic crystals (quasi-phase matched crystals) with domains of arbitrary sizes has been studied numerically. A simulated-annealing algorithm, working on the basis of numerical calculation, is developed to design quasi-phase matching gratings which can yield the desired amplitude and phase profile for second-harmonic pulses in the presence of pump depletion.
\end{abstract}

Keywords: second harmonic generation, quasi-phase matched crystals, nonlinear photonic crystals, pulse shaping.

Received: 2 February 2015

\section{Introduction}

Nonlinear Photonic Crystals (NPC) are of great interest because of their utility in practical applications, where it is necessary to control laser radiation. This interest in NPC's is especially warranted because they do not require the phase matching, which is necessary for uniform nonlinear crystals [1]. In such type of crystals, phase matching (or phase synchronism) of the interacting waves is obtained by periodically changing the sign of the second-order susceptibility, effectively widening the spectral range of frequency converters. Quasi-phase matched (QPM) or NPC gratings can also be used to provide dramatic pulse compression [2-3] and improved conversion efficiencies [4-5].

QPM gratings with a non-uniform periodicity can exhibit a longitudinally variable spectral response and entail the realization of advanced parametric processes [6], from highly efficient second harmonic generation (SHG) and parametric amplification in the case of a linear QPM chirp [4-5,7-9], to compression of second harmonic (SH) pulses when employing chirped fundamental-frequency (FF) pulses, [2-3, 10-11].

One of the interesting practical tasks, which can be solved by QPM gratings during SHG is the generation of SH pulses with arbitrarily chosen amplitude and phase profiles under the regime of pump depletion. A few methods have been developed to this end. Among them, the optimal control technique, based on Lagrange multipliers and real amplitudes [12-13], was applied to tailor ultra-short SH pulses by spatially varying the size of the nonlinearity. More recently, a similar approach was employed to design QPM gratings for picosecond SHG from femtosecond (fs) input FF pulses [14-19].

In this work, we discuss arbitrary fs pulse shaping based on SHG in engineered QPM in the regime of strongly depleted FF pump, taking into account both group velocity mismatch (GVM) and dispersion (GVD). To accomplish this, in contrast to previous work on the topic, we employ a simulated annealing algorithm (SAA), using simple fast Fourier 
transform and fourth-order Runge-Kutta algorithms. We improved the SAA, which was developed in previous work [18].

\section{Coupled-wave equations for SHG in arbitrary QPM gratings; numerical approach}

The slowly varying envelope equations describing pulse evolution under collinear frequency doubling in QPM are:

$$
\begin{aligned}
& \frac{\partial A_{1}}{\partial z}+\frac{1}{V_{1}} \frac{\partial A_{1}}{\partial t}-i \frac{\alpha_{1}}{2} \frac{\partial^{2} A_{1}}{\partial t^{2}}=-i \gamma_{1} \delta(z)\left(A_{1}\right)^{*} A_{2} \exp (-i \Delta k z) \\
& \frac{\partial A_{2}}{\partial z}+\frac{1}{V_{2}} \frac{\partial A_{2}}{\partial t}-i \frac{\alpha_{2}}{2} \frac{\partial^{2} A_{2}}{\partial t^{2}}=-i \gamma_{2} \delta(z)\left(A_{1}\right)^{2} \exp (i \Delta k z)
\end{aligned}
$$

with boundary conditions:

$$
\begin{aligned}
& \left.A_{1}(z, t)\right|_{z=0}=A_{o} \exp \left(-2 \ln 2(t / \tau)^{2}+i \varphi_{1}\right), \\
& \left.A_{2}(z, t)\right|_{z=0}=0
\end{aligned}
$$

where $A_{1}$ and $A_{2}$ are the complex amplitudes of FF and SH pulses, respectively; $A_{o}$ the peak amplitude of the FF excitation; $\mathrm{V}_{1}$ and $\alpha_{1}\left(\mathrm{~V}_{2}\right.$ and $\left.\alpha_{2}\right)$ the group velocity and the dispersive GVD spreading at FF (SH), respectively; $\tau$ input pulse duration (FWHM in intensity); $\gamma_{1}$ and $\gamma_{2}$ nonlinear coupling coefficients, with $\gamma \approx \gamma_{1} \approx \gamma_{2} \approx \frac{2 \pi}{\mathrm{n}\left(\omega_{o}\right) \lambda} d^{e f f} \approx \frac{2 \pi}{\mathrm{n}\left(2 \omega_{o}\right) \lambda} d^{e f f}$; $\mathrm{n}\left(\omega_{o}\right)$ and $\mathrm{n}\left(2 \omega_{o}\right)$ the refractive indices of $\mathrm{FF}$ and $\mathrm{SH}$ waves, respectively; $d^{\text {eff }}$ the effective nonlinearity $d^{e f f}=\chi^{(2)} / 2 ; \Delta k=2 k_{1}\left(\omega_{o}\right)-k_{2}\left(2 \omega_{o}\right)$ the phase-mismatch; $\varphi_{1}$ an initial FF phase and $\delta(\mathrm{z})$ the unitary sign-changing function defining the arbitrarily sized domains of the QPM grating (see Fig. 1).

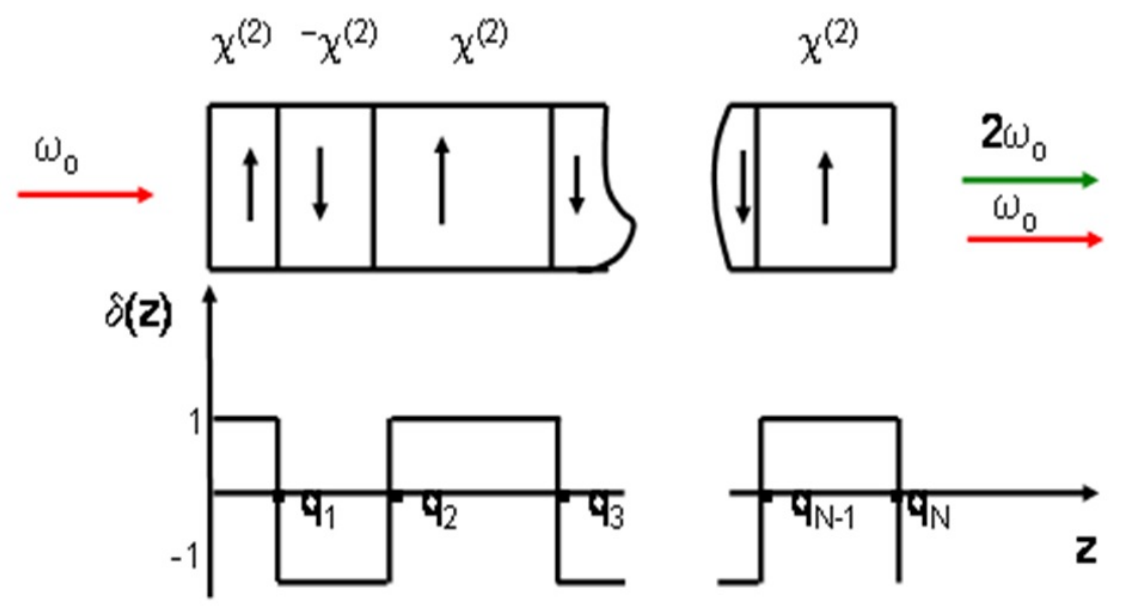

FIG. 1. Scheme of the arbitrary QPM grating, with $\delta(z)$ the dimensionless sign-changing aperiodic function of amplitude $|\delta(z)|=1$. The grating is comprised of $\mathrm{N}$ inverted domains with individual lengths $\mathrm{q}_{m}(1 \leqslant \mathrm{~m} \leqslant \mathrm{~N})$

Set (2) could be numerically integrated by various methods. The fast Fourier transform for the linear portion and the fourth-order Runge-Kutta (RK) method for the nonlinear regime ensure high accuracy and reduced iteration times. However, because of the aperiodic nature of $\delta(\xi)$, we resort to RK with variable integration steps $d \xi(m)$ ( $m$ is the domain number) inside each domain (see Fig. 2). GVM and GVD are accounted for by the fast Fourier transform. 

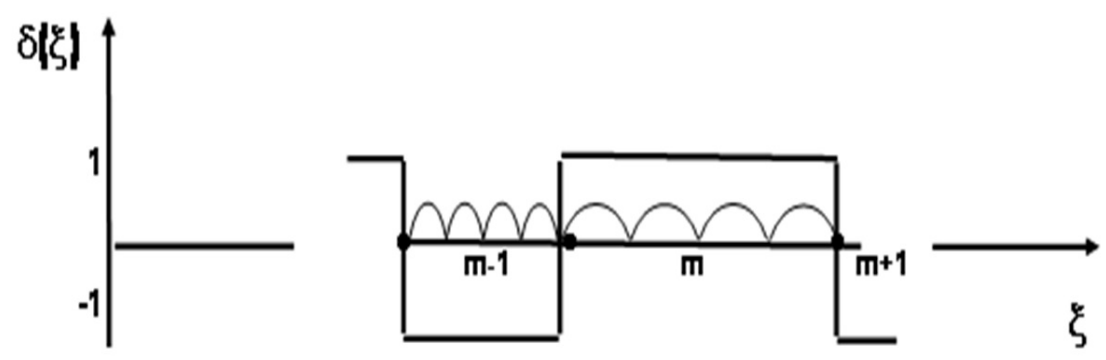

FIG. 2. Integration scheme. Here the number of steps in each domain is $j=4$

\section{Results}

We used SAA, which was developed earlier in [18]. This stems from the high accuracy of the PG-FROG traces used in calculating the RMS error [18]. Figure 3 shows the results obtained for a case of FF (1560 nm) for a $100 \mathrm{fs}$ FF to a Gaussian SH pulse with duration of 100 fs with a efficiency conversion of $20 \%$. Here and below, for all our calculations, we chose a $1 \mathrm{GW} / \mathrm{cm}^{2}$ peak intensity for $\mathrm{FF}$ time profile. However, for the domain size, we adopted a coarse resolution of $100 \mathrm{~nm}$, two orders of magnitude larger than what is achievable with the algorithm; we obtained an excellent convergence to the desired profile and conversion efficiency. Figure 3 (left) plots time profiles of FF (dotted) and desired (dashed) and calculated SH profiles (solid); Figure 3 (center) shows power evolutions of the interaction pulses in arbitrary designed QPM grating; Figure 3 (right) shows change of domain sizes as a function of their numbers.
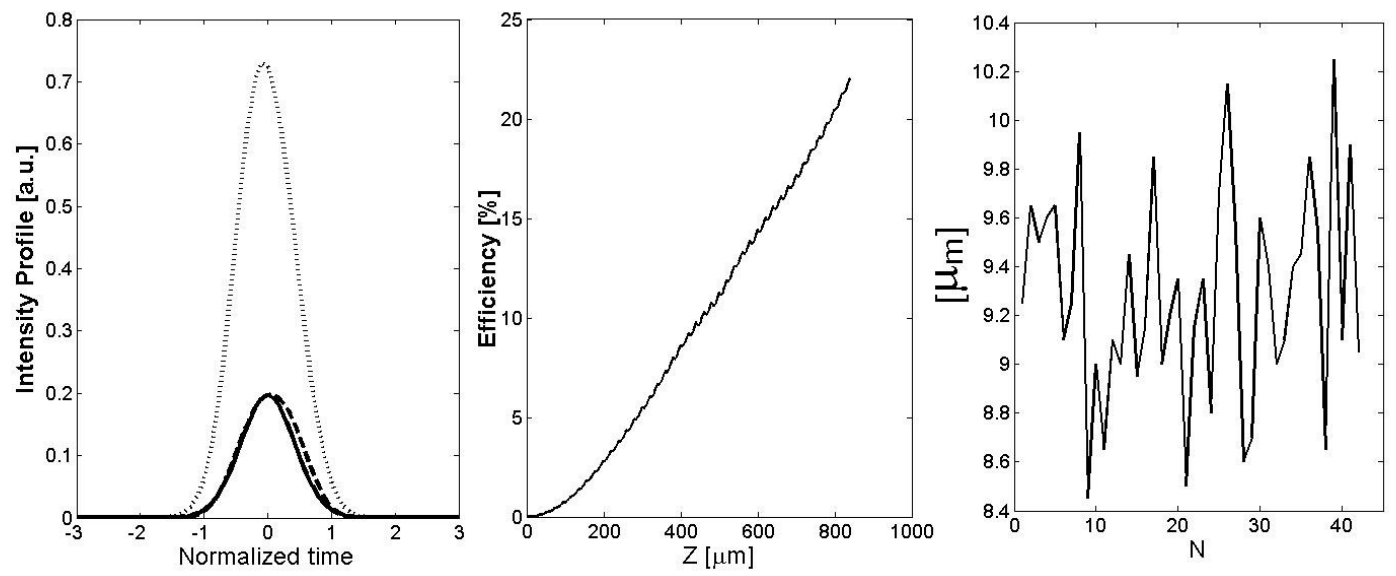

FIG. 3. Results of SAA algorithm for $100 \mathrm{fs}$ SH pulse with a $20 \%$ efficiency (center) for Lithium Niobate crystal at $1550 \mathrm{~nm}$ FF wavelength: (left) FF intensity distributions (doted), desired target SH profiles (dashed) and obtained SH profile (solid); (right) change of domain sizes as a function of their numbers

Noticeably, the required grating length increased as compared to a case, when we chose higher efficiency conversion (Fig. 4. shows these results). The grating is even longer than above; the agreement between the target and output pulses is quite satisfactory, despite the small but appreciable discrepancy between their PG-FROG traces. The results above demonstrate the good performance of the algorithm when simple Gaussian SH pulses are desired as the output, owing to the lack of sharp (temporal or spectral) features. Fig. 4. 
shows results for higher SH efficiency conversion ( $\sim 40 \%)$ other parameters with the same condition as Fig. 3. But here, the desired SH profile is chosen to be $150 \mathrm{fs}$.
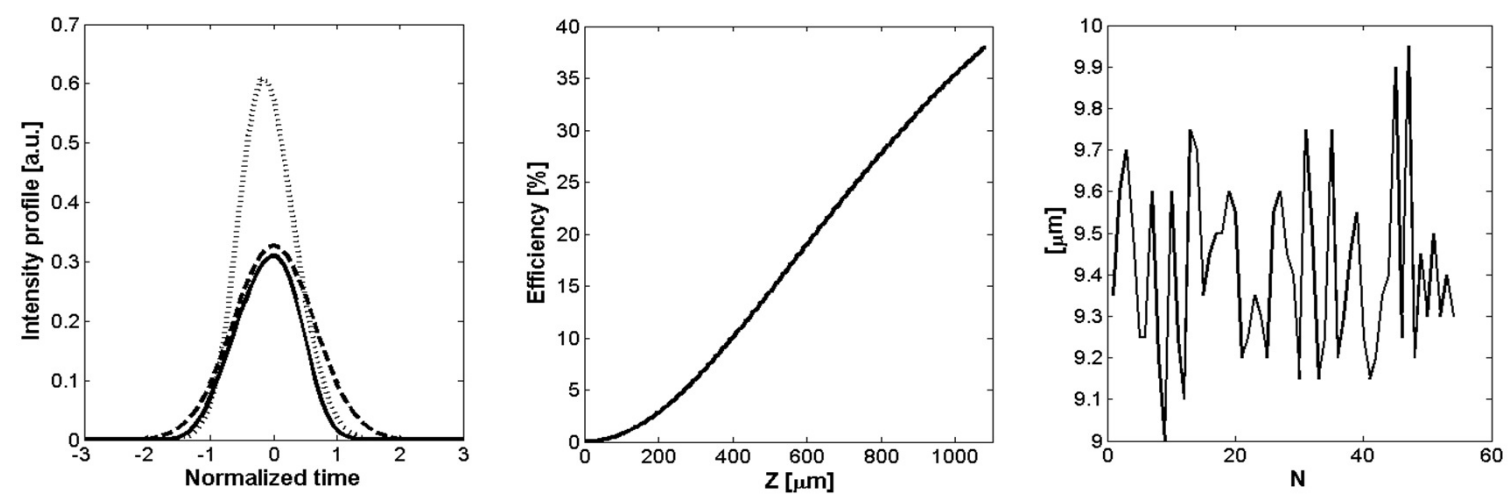

FIG. 4. Results for higher SH efficiency conversion (40\%). All curves are determined as Fig. 3. Here desired SH profile is chosen to be 150 fs

We also studied the chosen problem for shorter FF (50 fs) and SH (50 fs) pulses with $10 \%$ efficiency conversion. For this case, we could get results faster than previous cases as shown in Fig. 5. This results from the fact that shorter nonlinear QPM crystals generate shorter SH pulses, due to GVM between the interacting harmonics. For this we could initially get excellent results, and secondly, faster run times than in previous cases.
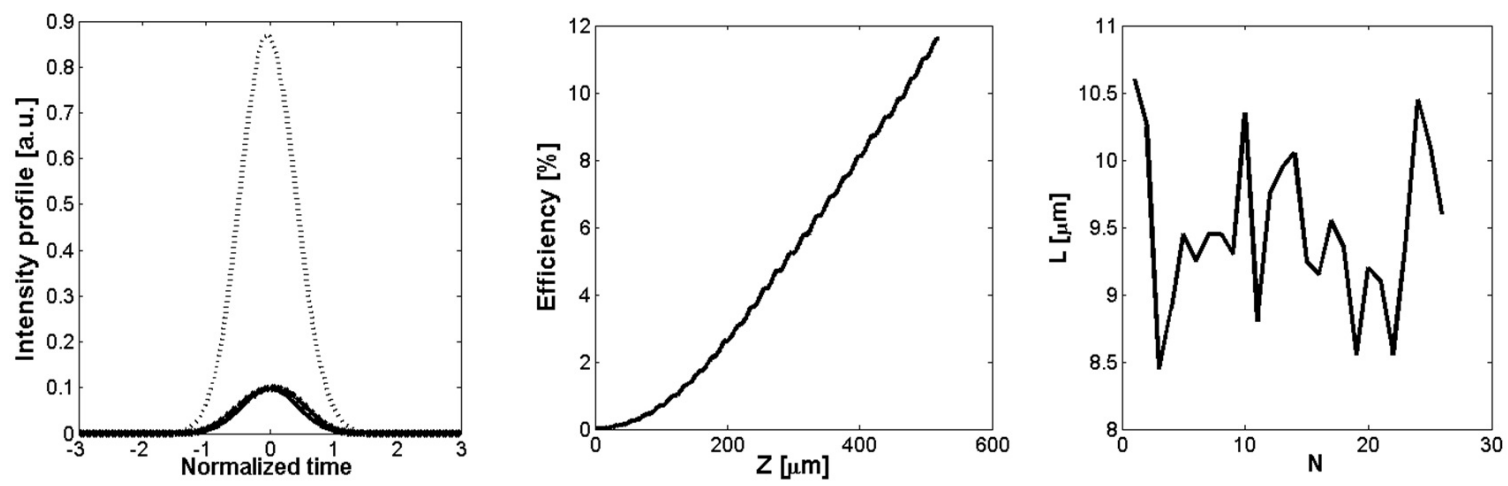

FIG. 5. Results for shorter FF (50 fs) and desired SH (50 fs) pulses with efficiency conversion of 10\%. All curves are determined as Fig. 3 and 4

It is necessary to note that for fs FF pulses it is easy to obtain the desired SH pulses of almost the same duration with high efficiency up to $50 \%$ (we did not present that result here). This is caused by smaller influence of GVM. But when we concentrate longer desired $\mathrm{SH}$ pulses than FF pulse, the codes run for a longer time and give worse results due to large number of domains. We believe this comes from our program interface, which was Matlab without parallel computing. So, if we could use some program interface with parallel computing, we could obtain excellent results more quickly for "heavy" target SH pulses.

\section{Conclusions}

In conclusion, we were able to design a variety of femtosecond pulse profiles through second-harmonic generation of $100 \mathrm{fs}$ and 50 fs Gaussian inputs at the FF using improved 
SAA. PG-FROG spectrograms were used to calculate RMS errors and lead to rapid convergence of the method with high accuracy. The results, outlined for the relevant case of an aperiodically poled Lithium Niobate crystal, demonstrate that proper engineering of a quasiphase-matched grating is feasible even under severe pump depletion and in the presence of limited fabrication resolution. The presented results can be used for obtaining femtosecond pulses with desired amplitude and phase profiles.

\section{References}

[1] Armstrong J. A., Bloembergen N., Ducuing J. and Pershan P. S. Interactions between Light Waves in a Nonlinear Dielectric. Physical Review, 127(5), P. 1918-1939 (1962).

[2] Arbore M. A., Marco O., Fejer M.M., Pulse compression during second-harmonic generation in aperiodic quasi-phase-matching gratings. Optics Letters, 22(12), P. 865-867 (1997).

[3] Arbore M.A., Galvanauskas A., Harter D., Chou M.H. and Fejer M.M. Engineerable compression of ultra-short pulses by use of second-harmonic generation in chirped-period-poled lithium niobate. Optics Letters, 22(17), P. 1341-1343 (1997).

[4] Artigas D., Reid D.T., Fejer M.M. and Torner L. Pulse compression and gain enhancement in a degenerate optical parametric amplifier based on aperiodically poled crystals. Optics Letters, 27(6), P. $442-444$ (2002).

[5] Artigas D., Reid D.T. Efficient femtosecond optical parametric oscillators based on aperiodically poled nonlinear crystals. Optics Letters, 27(10), P. 851-853 (2002).

[6] Fejer M.M., Magel G.A., Jundt D. H. and Byer R. L., Quasi-phase-matched second harmonic generation - Tuning and tolerances. IEEE J. Quantum Electron, 28(11), P. 2631-2653 (1992).

[7] Loza-Alvarez P., Reid D.T., Faller P., Ebrahimzadeh M. and Sibbett W., Simultaneous secondharmonic generation and femtosecond-pulse compression in aperiodically poled $\mathrm{KTiOPO}_{4}$ with a $\mathrm{RbTiOAsO}_{4}$-based optical parametric oscillator. J. Opt. Soc. Am. B, 16(9), P. 1553-1560 (1999).

[8] Loza-Alvarez P., Reid D. T., Faller P., Ebrahimzadeh M., Sibbett W., Karlsson H. and Laurell F. Simultaneous femtosecond-pulse compression and second-harmonic generation in aperiodically poled $\mathrm{KTiOPO}_{4}$. Optics Letters, 24(15), P. 1071-1073 (1999).

[9] Beddard T., Ebrahimzadeh M., Reid D.T. and Sibbett W. Five-optical-cycle pulse generation in the mid infrared from an optical parametric oscillator based on aperiodically poled lithium niobate. Optics Letters, 25(14), P. 1052-1054 (2000).

[10] Imeshev G., Arbore M.A., Fejer M.M., Galvanauskas A., Fermann M. and Harter D. Ultrashort-pulse second-harmonic generation with longitudinally nonuniform quasi-phase-matching gratings: pulse compression and shaping. J. Opt. Soc. Am. B, 17(2), P. 304-318 (2000).

[11] Imeshev G., Arbore M.A., Kasriel S. and Fejer M.M., Pulse shaping and compression by secondharmonic generation with quasi-phase-matching gratings in the presence of arbitrary dispersion. $J$. Opt. Soc. Am. B, 17(8), P. 1420-1437 (2000).

[12] Buffa R. Transient second-harmonic generation with spatially non-uniform nonlinear coefficients. $O p$ tics Letters, 27(12), P. 1058-1060 (2000).

[13] Buffa R. and Cavalieri S. Optimal control of type I second-harmonic generation with ultrashort laser pulses. J. Opt. Soc. Am. B, 17(11), P. 1901-1905 (2000).

[14] Conforti M., Baronio F. and Angelis C. De. From femtosecond infrared to picosecond visible pulses: temporal shaping with high efficiency conversion. Optics Letters, 32(13), P. 1779-1789 (2007).

[15] Reid D.T. Engineered quasi-phase-matching for second-harmonic generation. J. Opt. A: Pure Appl. Opt., 5, P. S97-S102 (2003).

[16] Sapaev U.K., Reid D.T. General second-harmonic pulse shaping in grating-engineered quasi-phasematched nonlinear crystals. Optics Express, 13(9), P. 3264-3276 (2005).

[17] Sapaev U.K. Optimum shaping of a spectral response of second harmonic generation process in the aperiodic quasi-phase matched nonlinear crystal. Optics Spectroscopy, 102(6), P. 1023-1027 (2007).

[18] Sapaev U.K., Assanto G. Femtosecond pulse synthesis by efficient second harmonic generation in engineered quasi phase matching gratings. Optics Express, 15(12), P. 7448-7457 (2007).

[19] Sapaev U.K., Yusupov D. B., Sherniyzov A.A., Uzakov A. A. Theory of backward second-harmonic generation of short laser pulses in periodically and aperiodically poled nonlinear crystals. Journal of Russian Laser Research, 33(2), P. 196-210 (2012). 